\title{
Damage Identification of Truss Structure Based on Improved BP Network
}

\author{
Jinsheng Fan ${ }^{1, a}$, Ying Yuan ${ }^{1, b}$ and Limei Zhang ${ }^{2, c}$ \\ ${ }^{1}$ Shijiazhuang University of Economics, Shijiazhuang,Hebei, 050031,China \\ ${ }^{2}$ Hebei University of Science and Technology, Shijiazhuang,Hebei, 050018,China \\ a490371584@qq.com, b275596117@qq.com, ${ }^{c} 123963769 @ q q . c o m$
}

\section{Keywords: Improved BP Network; Asymmetric truss; Damage Identification}

Abstract. Based on mode shape, a BP Network improved by using Fletcher-Reeves (FR) correction algorithm was put forward for identifying truss structure damages. Then through finite element analysis and calculating, the training and forecasting samples were got for the network. The trained BP neural network can identify the damage location and degree of the asymmetric truss structure. It proved that this method is practical and valid.

\section{Introduction}

In recent years, it's a development trend to build large and complex engineering structures, such as tall buildings, space grid structures, large span bridges, etc. Most of these buildings contain truss form. Their investment is huge, and they are often the places for people to gather or configure the important facilities, their security is not only to maintain the lives of thousands of people, but also has a major internationaland domestic impact. During the service period, due to the accidental load, fatigue load and overload, the individual components will be damaged, and it will even result in structural collapse. So the safety problem of these Structures must be the focus of our attention, it is very necessary to identify the structural damage timely and accurately and take corresponding maintenance measures to ensure the safety of large truss structures.

Structural damage identification problem is essentially a pattern classification problem. Neural network method has powerful parallel computing power, self-learning function, association ability and strong robustness. It has been widely used in pattern recognition, fault detection, non linear system identification and decision optimization, etc. Neural network is capable of dealing with the inherent mechanism of damage, it does not need the exact structure of the model, and it can easily handle the nonlinear damage mechanism. Because of the structure specific damage state can be expressed in the corresponding structural response mode, so the pattern recognition of neural network can be used to identify the structural damage .Many scholars have used network models to identify the structural damage, such as the BP neural network, the wavelet neural network, the radial basis function neural network, $[1,2,3]$, etc.

\section{The improved BP network}

BP neural network is widely used for structural damage diagnosis. BP network can realize nonlinear mapping between input and output parameters of structure system without the mathematical model, and the accuracy of the results of this mapping can be ensured by enough training samples. In this paper, the BP network is composed of three layers: input layer, hidden layer and output layer.

The traditional steepest descent BP algorithm is modified by the steepest descent direction along the gradient. Although the error rate is the fastest, but the speed of convergence is not necessarily the fastest, this paper uses the variable gradient algorithm to improve the BP network [4]. The first iteration of all the variable gradient method is carried out by the steepest descent direction of the gradient. Then it determines the linear search for optimal distance to carry on along the current search direction. It is shown in Eq. 1:

$$
x(k+1)=x(k)+o p(k)
$$


Where, $x(k)$ is the threshold vector between the layers for the $K$ iteration, $g(k)$ is the gradient vector of the output error of the neural network for the $K$ iteration, $\alpha$ is the learning rate, and it is a training constant, $p(k)$ is the search direction for the $k+1$ iteration,. It is shown in Eq. 2:

$$
p(k)=-g(k)+\beta(k) p(k-1)
$$

$\beta(k)$ is obtained by Fletcher-Reeves(FR) correction algorithm. It is shown in Eq. 3:

$$
\beta(k)=\frac{g^{T}(k) g(k)}{g^{T}(k-1) g(k-1)}
$$

The rate of this FR algorithm is usually much faster than variable learning speed algorithm, and its required storage space is only little more than the ordinary algorithm.

\section{Selection of damage identification parameters}

For the damage identification of structures, it will directly affect the size and accuracy of the workload because of the parameter selection. In this paper, the sensitivity and applicability of a variety of characteristic parameters were analyzed and compared to choose the better one for truss structural damage. Then modal curvature ratio (MCR) [5] was initially selected as the feature parameter.

Firstly use the central difference formula to obtain the structural modal curvature approximately. It is shown in Eq. 4:

$$
\phi_{i, j}^{\prime \prime}=\left(\phi_{i+1, j}-2 \phi_{i, j}+\phi_{i-1, j}\right) / h^{2}
$$

Where, $\phi_{i, j}$ is displacement amplitude of the calculating node, $\phi_{i+1, j}, \phi_{i-1, j}$ are respectively the displacement amplitude of its adjacent points, $i$ is the node number, $j$ is modal order, $h$ is the distance between two nodes, namely the element length.

Then MCR is calculated shown in Eq. 5:

$$
\gamma=\phi_{d i, j}^{\prime \prime} / \phi_{i, j}^{\prime \prime}
$$

Where, $\phi_{i, j}^{\prime \prime}, \phi_{d i, j}^{\prime \prime}$ are respectively modal curvature of the calculating nodes before and after damage. At the location of structural damage, as a result of modal curvature change, great change must take place on MCR. Following a numerical model of asymmetric truss was set up by ANSYS to verify the validity of the parameter. Its dynamic behavior is more complex than a symmetric truss.

The model is a 5 spans asymmetric steel truss in fig. 1 , its length is $15 \mathrm{~m}$, each segment is $3 \mathrm{~m}$, its oblique abdominal rod is $4.24 \mathrm{~m}$, its rods are I-shaped steel sections and the cross section is $0.00025 \mathrm{~m}^{2}$, elastic modulus is $2.0^{*} 10^{11} \mathrm{pa}$, density is $7850 \mathrm{~kg} / \mathrm{m}^{3}$, Poisson's ratio is 0.27 . All of the rods are connected by hinges, a total of 10 nodes, 17 elements. The top and bottom rods are simulated by Beam4; web members are simulated by Link8. It is thought that in modal analysis, the damage will only reduce element stiffness and mass does not change, so it only use the reduction of elastic modulus $E$ to simulate the element damage, the different values of $E$ stand for the different stiffness. The following is the part of the Analysis of the calculation results. The first order modal vibration mode is taken in the calculation.

In fig. 2, the sample simulated is that element 8 different degree damages occur. It can be seen that only the MCRs of node 4 and 5 associated with element 8 change suddenly. Others basically have no change. And the more serious the damage, the more obvious the change. 

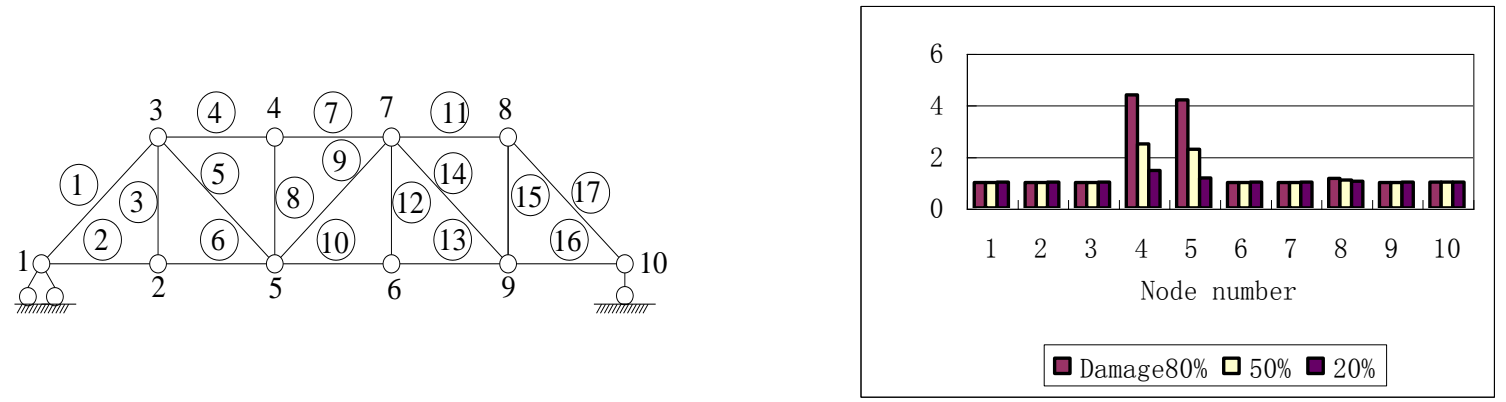

Fig.1 Asymmetric truss structure model Fig.2 Mode shape curvature ratio as element 8 damaged

From above it can draw a conclusion that by the MSCR it can identify truss structural damage location and the damage degree. So it is a good parameter for damage identification.

\section{The construction of BP network and truss structure damage identification}

Following, a BP neural network was created to identify the damage of the truss structure shown in fig. 1. The network has three layers: they are an input layer, a hidden layer and an output layer. It mainly used the neural network toolbox newff function to build the network model[6]: Net=newff(minmax $(\mathrm{P}),[21,17]$, \{'tansig', 'logsig' \}, 'traingfr'). Traingfr is the training function founded according to the Fletcher-Reeves(FR) correction algorithm.

There are 10 neurons on the network's input layer as the MCRs of various nodes, 17 neurons are on output layer as the damage degrees of every element. If an output layer neurons has a value of 0 , it means no damage.

Table 1 Identification results of various damage Samples

\begin{tabular}{ccccccc}
\hline \multirow{2}{*}{$\begin{array}{c}\text { Element } \\
\text { number }\end{array}$} & \multicolumn{2}{c}{ Sample 1 } & \multicolumn{2}{c}{ Sample 2 } & \multicolumn{2}{c}{ Sample 3 } \\
Output & $\begin{array}{c}\text { Expected } \\
\text { value }\end{array}$ & $\begin{array}{c}\text { Output } \\
\text { value }\end{array}$ & $\begin{array}{c}\text { Expected } \\
\text { value }\end{array}$ & $\begin{array}{c}\text { Output } \\
\text { value }\end{array}$ & $\begin{array}{c}\text { Expected } \\
\text { value }\end{array}$ \\
\hline 1 & 0 & 0 & 0.0001 & 0 & 0.0167 & 0 \\
2 & 0 & 0 & 0.0003 & 0 & 0.0056 & 0 \\
3 & 0.0002 & 0 & 0.0001 & 0 & 0.0096 & 0 \\
4 & 0 & 0 & 0.0036 & 0 & $\underline{0.6642}$ & $\underline{0.65}$ \\
5 & 0.0006 & 0 & 0.0012 & 0 & 0.0206 & 0 \\
6 & 0.0008 & 0 & 0.0025 & 0 & 0.0025 & 0 \\
7 & 0.0016 & 0 & $\underline{0.1732}$ & $\underline{0.15}$ & 0.0232 & 0 \\
8 & 0.0019 & 0 & 0.0053 & 0 & 0.0227 & 0 \\
9 & $\underline{0.2021}$ & $\underline{0.2}$ & 0.0062 & 0 & 0.0066 & 0 \\
10 & 0.0017 & 0 & 0.0029 & 0 & 0.0054 & 0 \\
11 & 0.0005 & 0 & 0.0058 & 0 & 0.0162 & 0 \\
12 & 0.0022 & 0 & 0.0044 & 0 & 0.0082 & 0 \\
13 & 0 & 0 & 0.0024 & 0 & 0.0176 & 0 \\
14 & 0.0006 & 0 & 0.0583 & 0 & 0.0215 & 0 \\
15 & 0 & 0 & 0.0083 & 0 & $\underline{0.2816}$ & $\underline{0.25}$ \\
16 & 0 & 0 & $\underline{0.4225}$ & $\underline{0.45}$ & 0.0138 & 0 \\
17 & 0 & 0 & 0.0147 & 0 & 0.0062 & 0 \\
\hline
\end{tabular}


Using the above numerical calculation model, the structural modal shape before and after the damage was analyzed by ANSYS software, then the displacement of the first order modal shape was extracted. By these data, all the samples of single and two different degrees element damages in the structure were calculated. Then the curvature mode shapes of nodes were obtained. With the elastic modulus of 17 elements reduced by $5 \%, 30 \%, 55 \%, 75 \%, 90 \%$, a total of 1445 samples were obtained to train the network. The training time was cut by $30 \%$ using FR algorithm. Then 3 forecasting samples were used to test the effect of the trained network. The test results are shown in Table 1.

From the results in Table 1, the output values of the neurons corresponding to health elements are very close to 0 , so the neural network can accurately identify the damage location. For predicting damage degree, the maximum absolute error is $5.83 \%$ in the Sample 2. The reason is that both the nodes of element 14 are included in the damaged elements, so it is considered to be damaged, except this element, the others absolute error is not beyond $3.16 \%$. From the overall, damage identification error is within the scope of the permit, the results are acceptable.

\section{Conclusion}

(1) From the simulation example, it can see that for the asymmetric truss structure the MCR of the nodes can be used as characteristic parameter to identify its damage. And it can calculate MCR only needing the first order vibration mode. This greatly increases the practicability of the method.

(2) Improved by FR correction algorithm, the BP network's training time is greatly shortened, and the neural network can better predict the damage location and degree of truss structure.

\section{Acknowledgements}

This work was financially supported by Natural Guidance Project of Hebei Province Department of Education (Z2012137).

\section{References}

[1] S.W.Chen and G.Q.Li: J.Earthquake engineering and engineering vibration In Chinese. Vol.22 (2002), P.18

[2] H.Pan and Y.Huang:.J.Computer engineering and applications In Chinese. Vol. 46 (2010), P.204

[3] W.B.Rao and D.H.Wu: J.Solid mechanics In Chinese. Vol.23 (2002), P.477

[4] Pin Zhou: MATLAB neural network design and application (Tsinghua university press, China 2013)

[5] J.S.Fan,Y.Z.Su and X.F.Li:J.Shanxi Architecture In Chinese. Vol.33 (2007), P.70

[6] Feisi science and technology product research and development center: MATLAB6.5 auxiliary neural network analysis and design (Electronic industry press, China 2003) 